\title{
Sequências de Baixa Discrepância Aplicadas à Avaliação de Qualidade de Imagens Comprimidas
}

E.A. LIMA ${ }^{1}$, F. MADEIRO ${ }^{2}$, Universidade Católica de Pernambuco, 50050-900 Recife, PE, Brasil.

Resumo. Um dos desafios em codificação de imagens é a concepção de sistemáticas eficientes para a avaliação de qualidade de imagens reconstruídas. Este trabalho apresenta uma abordagem para a avaliação de qualidade de imagens baseada em sequências de baixa discrepância. Resultados obtidos para imagens $256 \times 256$ submetidas à quantização vetorial mostram que, com apenas $7 \%$ dos pixels, o erro médio quadrático calculado com a abordagem proposta difere em menos de $5 \%$ do valor exato desta métrica, calculado usando a totalidade dos pixels.

Palavras-chave. Processamento e transmissão de imagens, medidas de qualidade de imagens, sequências de baixa discrepância.

\section{Introdução}

A compressão de sinais, cujo objetivo fundamental é reduzir o número de bits necessários para representar adequadamente os sinais (voz, imagem, áudio, vídeo), desempenha um papel importante em aplicações que necessitam minimização dos requisitos de largura de faixa e/ou de capacidade de armazenamento, tais como: sistemas multimídia, redes digitais de serviços integrados, videoconferência, sistemas de resposta vocal, correio de voz (voicemail), difusão de música, facsímile de alta resolução, televisão de alta definição, telefonia móvel, sistemas de armazenamento de imagens médicas e de impressões digitais e transmissão de imagens de sensoriamento remoto obtidas por satélites.

Apesar de alguns sistemas não apresentarem grandes limitações de largura de faixa, como é o caso das redes de comunicações por fibra ótica, e embora a evolução tecnológica esteja continuamente contribuindo para o surgimento de memórias com grande capacidade de armazenamento, a compressão de sinais desempenha um papel importante, devido a uma série de fatores, tais como: a grande utilização dos sistemas multimídia tem levado ao aumento da demanda de armazenamento de voz, música, imagens, vídeo e dados em forma comprimida; um maior número de canais de comunicação pode ser multiplexado em sistemas de faixa larga, por meio do uso de técnicas de compressão para reduzir os requisitos de largura de faixa de cada

\footnotetext{
${ }^{1}$ eal@dei.unicap.br

2madeiro@dei.unicap.br
} 
sinal a ser multiplexado; nas redes digitais de serviços integrados (ISDN, integrated services digital networks), as técnicas de compressão permitem uma integração eficiente de sinais e dados.

Codificação de sinal é o processo de representar um sinal de informação de tal maneira a atingir um objetivo de comunicação desejado, tal como conversão analógico-digital, transmissão a baixas taxas de bits ou criptografia de mensagens. $\mathrm{Na}$ literatura, os termos codificação de fonte e compressão de sinais têm sido empregados a técnicas para obtenção de uma representação digital comprimida de um sinal [8].

Um dos grandes desafios em codificação digital de sinais é a concepção e o desenvolvimento de metodologias de avaliação de qualidade de sinais reconstruídos (obtidos com a aplicação de técnicas de compressão). De forma geral, as medidas utilizadas para avaliação da qualidade de sinais enquadram-se em duas classes: medidas de qualidade subjetivas e medidas de qualidade objetivas. As primeiras baseiam-se em comparações (realizadas por meio de testes de escuta ou de visualização), entre o sinal original e o sinal processado, realizadas por um grupo de pessoas, que subjetivamente classificam a qualidade do sinal processado segundo uma escala pré-determinada. As medidas objetivas, por sua vez, baseiam-se numa comparação matemática direta entre os sinais original e processado [2].

Para serem úteis, as medidas de qualidade objetivas devem apresentar, no mínimo, duas características. Primeiramente, devem ter significado subjetivo, no sentido de que pequenas e grandes variações das medidas objetivas devem significar pequenas e grandes variações da qualidade subjetiva dos sinais reconstruídos respectivamente devem apresentar, portanto, uma correlação forte, positiva ou negativa, com resultados de avaliação subjetiva. Segundo, devem ser matematicamente tratáveis e facilmente implementáveis.

Medidas de qualidade subjetivas são utilizadas para avaliar de forma plena, definitiva, a qualidade de algoritmos/técnicas de codificação de sinais. Os testes subjetivos, contudo, são de difícil realização, uma vez que requerem a disponibilidade de um grande número de avaliadores (entre leigos, especialistas e possíveis usuários do sistema de codificação de sinais), envolvem grande volume de sinal processado e necessitam da disponibilidade de laboratórios com condições adequadas ao desenvolvimento das avaliações subjetivas, enfim, são bastante dispendiosos de tempo, implicando custo elevado de realização. Devido a esses problemas, as medidas de qualidade objetivas, por serem pouco dispendiosas de tempo, desempenham um papel importante no processo de avaliação de qualidade de sinais reconstruídos e constituem ferramenta valiosa no processo de ajuste de parâmetros de algoritmos/técnicas de compressão.

O presente trabalho apresenta uma abordagem para a avaliação de qualidade objetiva de imagens baseada em sequências de baixa discrepância. O alvo é selecionar uma parcela dos pixels, de tal forma que a medida de qualidade calculada com a parcela de pixels seja próxima da medida de qualidade calculada com a imagem completa (com a totalidade dos pixels). São apresentados resultados referentes à quantização, que desempenha papel importante em sistemas e algoritmos de compressão de imagens (como, por exemplo, JPEG - joint photographics experts group), levando a taxas de compressão obtidas à custa de degradações irreversíveis nas ima- 
gens. Os resultados obtidos para imagens submetidas à quantização vetorial $[5,6]$ mostram que, com apenas $7 \%$ dos pixels de imagens $256 \times 256$, o valor encontrado para o MSE (mean square error) difere em menos de $5 \%$ do valor real desta métrica, calculada usando a totalidade dos pixels das imagens.

\section{Avaliação de Qualidade Objetiva de Imagens}

Dentre as medidas objetivas utilizadas para avaliação de qualidade de imagens, podem ser citadas [4]: diferença média, conteúdo estrutural, correlação cruzada normalizada, qualidade de correlação, diferença máxima, fidelidade de imagem, norma $L_{p}$, erro absoluto normalizado. Duas medidas objetivas amplamente utilizadas para a avaliação de qualidade de imagens são a relação sinal-ruído de pico (PSNR, peak signal to noise ratio) e o erro médio quadrático (MSE, mean square error). A primeira é definida como 10 vezes o logaritmo na base 10 da razão entre o quadrado do valor de pico da amplitude do sinal de entrada, $v_{p}^{2}$, e o erro médio quadrático:

$$
\mathrm{PSNR}=10 \log _{10}\left[\frac{v_{p}^{2}}{\mathrm{MSE}}\right]
$$

Para o caso de uma imagem original codificada a 8,0 bpp (256 níveis de cinza), $v_{p}=255$. O erro médio quadrático entre as imagens original e reconstruída é definido como

$$
\mathrm{MSE}=\frac{1}{L \cdot C} \sum_{l=1}^{L} \sum_{c=1}^{C}[F(l, c)-\widehat{F}(l, c)]^{2},
$$

em que $F(l, c)$ e $\widehat{F}(l, c)$ representam os valores de pixels das imagens original e reconstruída, $l$ designa a $l$-ésima linha e $c$ denota a $c$-ésima coluna de uma imagem (matriz) $L \times C$.

A avaliação de qualidade de imagem tem sido objeto de estudo de diversas pesquisas, tendo sido abordada suscinta ou profundamente em diversos trabalhos (e.g. [1, $3,4,11,14])$.

\section{Sequências de Baixa Discrepância}

Intuitivamente, uma sequência $\left\{x_{1}, x_{2}, \cdots\right\}$ de números reais no intervalo $I=[0,1]$ é dita uniformemente distribuída quando qualquer subintervalo de comprimento $L$ do intervalo $[0,1]$ contém aproximadamente o mesmo percentual de pontos da sequência e tal percentual é proporcional ao comprimento $L$.

Em termos mais precisos, dados $N$ inteiro e $E \subseteq[0,1]$, definimos a função de contagem $A(E ; N)=\left|\left\{1 \leq n \leq N: x_{n} \in E\right\}\right|$, i.e, a quantidade, dentre os primeiros $N$ termos da sequência, que são elementos de $E$. Uma sequência $\left\{x_{n}\right\}_{n=1}^{\infty} \subseteq I$ é definida como uniformemente distribuída (no intervalo $[0,1]$ ) se

$$
\forall a, b \in \mathbb{R}, 0 \leq a<b \leq 1 \text { temos } \lim _{N \rightarrow \infty} \frac{A([a, b) ; N)}{N}=b-a .
$$


Tal definição é facilmente estendida para sequências multidimensionais ( [9] pp 47).

O conceito de discrepância mede o quanto uma dada sequência difere de uma sequência uniformemente distribuída. Formalmente, dada uma sequência finita $\left\{x_{1}\right.$, $\left.x_{2}, \cdots, x_{N}\right\}$, sua discrepância é definida como o valor

$$
D_{N}=\sup _{0 \leq a<b \leq 1}\left|\frac{A([a, b) ; N)}{N}-(b-a)\right| .
$$

Uma sequência infinita $\left\{x_{n}\right\}_{n=1}^{\infty} \subseteq I$ é dita de baixa discrepância se $\lim _{N \rightarrow \infty} D_{N}$ $=0$, i.e, uma sequência é de baixa discrepância quanto tende a uma sequência uniformemente distribuída. Assim como na definição de uniformidade, a definição de discrepância é imediatamente generalizada para sequências multidimensionais ( [9] pp 92 e seguintes).

A importância do conceito de discrepância na aproximação dos valores numéricos de integrais definidas pode ser ilustrada pela versão fraca da desigualdade de Koksma (conforme [9] pp 143, Teorema 5.1).

Teorema 3.1. Seja $f: I \rightarrow \mathbb{R}$ contínua e $\left\{x_{1}, \cdots, x_{N}\right\} \subseteq I$ um conjunto de pontos de discrepância $D_{N}$, então

$$
\left|\frac{1}{N} \sum_{n=1}^{N} f\left(x_{n}\right)-\int_{0}^{1} f(t) d t\right| \leq D_{N} \max _{t \in I}|f(t)| .
$$

Portanto, quanto mais rápida for a convergência de uma sequência de baixa discrepância, menor o erro cometido na estimativa de uma integral utilizando um subconjunto de pontos da mesma.

Existem diversos exemplos de sequências de baixa discrepância na literatura. Uma das mais conhecidas é a sequência proposta por Van der Corput [13] e generalizada para o caso multidimensional por Halton [7]. Para tal sequência, vale o resultado $D_{N} \leq \frac{\log (N+1)}{N \log (2)}$, o que torna a discrepância de tal sequência uma das de mais rápida convergência conhecidas. Formalmente, definimos a sequência de Van der Corput-Halton de dimensão $k$ como a sequência formada pelos pontos

$$
\left(\phi_{m_{1}}(n), \phi_{m_{2}}(n), \cdots, \phi_{m_{k}}(n)\right), n=1,2, \cdots,
$$

em que $m_{1}, m_{2}, \cdots, m_{k}$ são relativamente primos dois-a-dois e

$$
\phi_{m}(n)=\sum_{j=0}^{s} a_{j} m^{-j-1},
$$

com $n-1=\sum_{j=0}^{s} a_{j} m^{j}$ a expansão de $n-1$ na base $m_{j}$. Se os números $m_{i}$ são tomados como a sequência dos números primos, i.e., $m_{1}=2, m_{2}=3, m_{3}=5, \cdots$, a sequência obtida é chamada de sequência clássica de Van der Corput-Halton.

\subsection{Aplicações de sequências de Baixa Discrepância na Ava- liação da Qualidade de Imagens}

Sejam $F(l, c)$ e $\widehat{F}(l, c)$ os valores de pixels das imagens original e reconstruída, em que $l$ designa a $l$-ésima linha e $c$ denota a $c$-ésima coluna de uma imagem (matriz) 
$L \times C$. Seja $S=\left\{\left(x_{i}, y_{i}\right)\right\}_{i=1}^{\infty}$ uma sequência qualquer de pontos de coordenadas inteiras contidas no intervalo $[1, L] \times[1, C]$. Podemos determinar os valores de nível de cinza correspondentes nas imagens original e reconstruída referentes a um ponto em particular $p_{j}=\left(x_{j}, y_{j}\right), 1 \leq j \leq k$ da sequência, ou seja, $F\left(p_{j}\right)=F\left(x_{j}, y_{j}\right)$ e $\widehat{F}\left(p_{j}\right)=\widehat{F}\left(x_{j}, y_{j}\right)$. Definimos uma estimativa $\widehat{M S E}_{S}$ do valor exato da métrica MSE (definida na Equação 2.2) na sequência $S$ como o valor

$$
\widehat{M S E}_{S}(k)=\frac{1}{k} \sum_{j=1}^{k}\left[F\left(p_{j}\right)-\widehat{F}\left(p_{j}\right)\right]^{2} .
$$

A escolha de $S$ como uma sequência de pontos aleatórios tem por vantagem o fato de a cobertura da imagem promovida pelos pontos da sequência não ser fixada. Infelizmente, o custo computacional de gerar tal sequência tende a ser elevado, uma vez que, para a obtenção de diferentes estimativas de $M S E$, é necessária a geração de diferentes sequências a fim de manter o caráter aleatório da escolha dos pontos. As Figuras 1(a) e 1(c) ilustram a cobertura promovida pela escolha de 100 e de 1.000 pontos aleatórios em uma matriz de tamanho $256 \times 256$. Observe que os pontos escolhidos na primeira amostragem ${ }^{3}$ (100 pontos) não formam, necessariamente, um subconjunto do conjunto obtido na segunda amostragem (1.000 pontos).

Uma maneira de obter uma estimativa com uma sequência computacionalmente simples do valor de $M S E$ seria tomar uma amostragem uniforme de um subconjunto dos $L C$ pontos (pixels) da imagem. A desvantagem desta abordagem é que, se forem gerados $\lambda$ pontos que contemplam uma cobertura uniforme, então não é possível aproveitar esses mesmos $\lambda$ pontos para que sejam gerados $\gamma>\lambda$ pontos ainda com cobertura uniforme.

Uma maneira de combinar os aspectos desejados de cobertura uniforme - em todos os estágios da geração da sequência - presentes na escolha de $S$ como uma sequência aleatória com o baixo custo computacional de uma amostragem uniforme simples é amostrar $S$ como uma sequência de baixa discrepância no conjuntos dos $L C$ pontos da imagem. Isto ocorre pois a sequência de baixa discrepância é pseudoaleatória, assim, é necessário gerá-la uma única vez e mantê-la em arquivo que pode ser usado em todos os experimentos em seguida, de forma que seu custo computacional é $\mathcal{O}(1)$. Mais ainda, apesar de ser uma sequência pseudo-aleatória, uma sequência de baixa discrepância tem por principal propriedade a manutenção de uma cobertura aproximadamente uniforme em todos os estágios de amostragem. As Figuras 1(b) e 1(d) ilustram a cobertura promovida pela escolha de 100 e de 1.000 pontos em uma matriz de tamanho $256 \times 256$. Observe que os pontos escolhidos na primeira amostragem (100 pontos) formam um subconjunto do conjunto obtido na segunda amostragem (1.000 pontos), de maneira que o mesmo arquivo de pontos foi utilizado em ambos (um arquivo de $256 \times 256$ pontos é gerado uma única vez, uma amostragem particular de tamanho $k$ é feita simplemente tomando os $k$ primeiros pontos do arquivo).

A escolha de $S$ como uma sequência de baixa discrepância permite, pelo uso da versão discreta do Teorema de Koksma (Teorema 3.1), estimar o erro obtido

\footnotetext{
${ }^{3}$ Nesta e em outras passagens do artigo, usa-se o termo amostragem no sentido de seleção, e não em termos do processo de amostragem segundo o Teorema de Nyquist.
} 


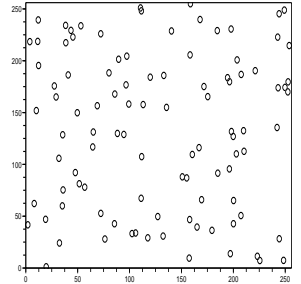

(a) 100 pontos aleatórios

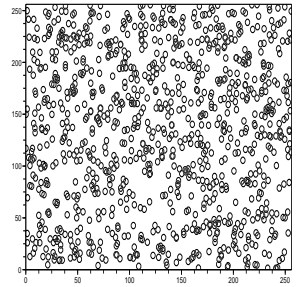

(c) 1.000 pontos aleatórios

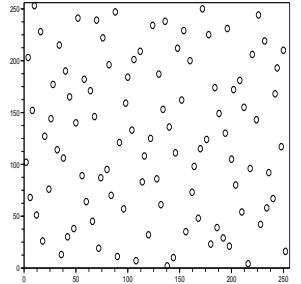

(b) 100 pontos baixa discrepância

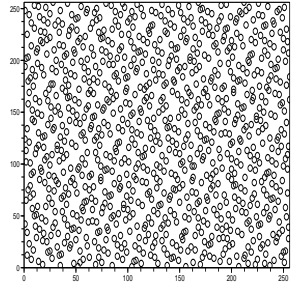

(d) 1.000 pontos baixa discrepância

Figura 1: Cobertura em pixels de uma região $256 \times 256$ promovida (à esquerda) por uma escolha aleatória de pontos e (à direita) utilizando a sequência de Van Der Corput-Halton. Observe a formação de áreas mais densas na escolha de pontos aleatórios, implicando uma cobertura menos uniforme da imagem.

por uma dada amostragem. No presente trabalho, $S$ foi tomado como a sequência de Van der Corput-Halton. Para esta escolha, a estimativa $\widehat{M S E}_{S}(k)$ é denotada simplesmente por $\widehat{M S E}(k)$.

\section{Resultados}

Foram testadas as imagens clássicas Barbara, Boat, Clock, Elaine, Goldhill, Lena, Mandrill, Peppers e Tiffany codificadas no formato PGM (Portable Graymap Format) com 256 níveis de cinza ( 8 bits por pixel). As imagens estão apresentadas na Figura 2.

As simulações realizadas no presente trabalho contemplaram imagens reconstruídas com uso da técnica de quantização vetorial $(\mathrm{QV})$ para o propósito de compressão. A QV consiste em mapear (quantizar) blocos de $K$ pixels da imagem original em um subconjunto finito do espaço euclidiano $K$-dimensional, $\mathcal{R}^{K}$, denominado dicionário. A taxa de codificação da QV, expressa em bits por pixel (bpp), é dada por $R=\frac{1}{K} \log _{2} N$, em que $N$ é o tamanho do dicionário (ou número de vetores de reconstrução, número de vetores-código). Neste trabalho, foram utilizados blocos de $4 \times 4$ pixels (QV com dimensão $K=16$ ) e tamanhos de dicionário $N=$ 


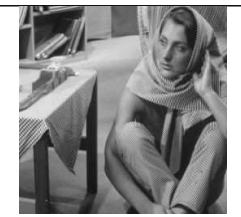

(a) Barbara.

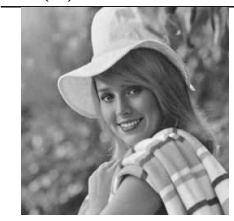

(d) Elaine.

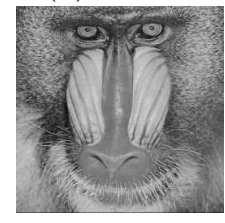

(g) Mandrill.

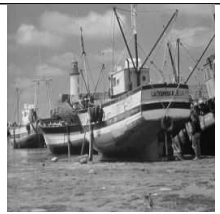

(b) Boat.

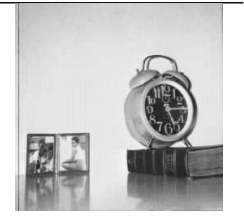

(c) Clock.

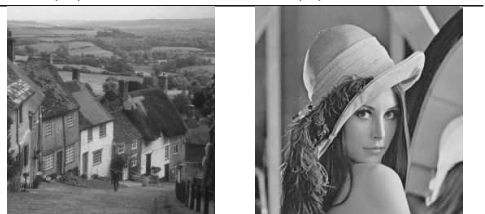

(e) Goldhill.

(f) Lena.

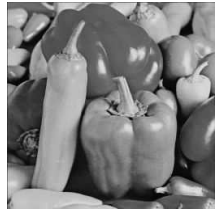

(h) Peppers.

(i) Tiffany.

Figura 2: Imagens $256 \times 256$ usadas como conjunto de testes.

$32,64,128,256$ e 512 . Portanto, as taxas de codificação consideradas variaram de 0,3125 bpp a 0,5625 bpp.

A medida de qualidade objetiva considerada é o MSE, calculado considerando a imagem original (codificada a $8 \mathrm{bpp}$ ) e a imagem reconstruída (submetida à quantização vetorial, com taxa de codificação $\left.R=\frac{1}{K} \log _{2} N\right)$. São apresentados resultados de MSE calculados considerando a totalidade dos pixels $(256 \times 256)$ das imagens original e reconstruída (MSE convencional) e resultados de MSE considerando uma parcela de pixels das imagens, obtida por meio de sequência de baixa discrepância. São apresentados resultados concernentes à diferença máxima que se obtém entre o MSE convencional e a estimativa de MSE via sequência de baixa discrepância ao ser utilizado um número de pontos igual ou superior a uma parcela de pontos obtida pelo método de Van Der Corput-Halton.

Nas estimativas do valor de MSE foram utilizados subconjuntos da sequência clássica de Van Der Corput-Halton bidimensional. Denotando $V D H=\left\{\left(x_{1}, y_{1}\right)\right.$, $\left.\left(x_{2}, y_{2}\right), \ldots\right\}$ a sequência de Van Der Corput-Halton bidimensional escalonada, o valor estimado do MSE pelos primeiros $k$ pontos da sequência $V D H$ é definido como

$$
\widehat{M S E}_{k}=\frac{1}{k} \sum_{i=1}^{k}\left[F\left(x_{i}, y_{i}\right)-\widehat{F}\left(x_{i}, y_{i}\right)\right]^{2},
$$

i.e, a soma dos quadrados das diferenças dos $k$ primeiros pontos da sequência $V D H$. Observe que, independentemente das imagens a serem avaliadas, os pontos escolhidos de avaliação serão sempre os mesmos, o que poderia sugerir uma dependência 
entre as estimativas obtidas e os diferentes tipos de imagens a serem avaliadas. Os resultados obtidos, contudo, indicam não ser este o caso. De fato, o uso generalizado de sequências de baixa discrepância no cálculo numérico de integrais multidimensionais ilustra que, em casos práticos, tal dependência parece não ser relevante [12].

O erro percentual de uma estimativa com relação ao valor exato da métrica é dado por

$$
\text { erro }_{k}=\max _{k \leq i \leq m} \frac{\left|\widehat{M S E}_{i}-M S E\right|}{M S E},
$$

ou seja, o erro define o menor intervalo centrado no valor exato da métrica que contém todas as estimativas que podem ser obtidas utilizando uma quantidade de pontos maior ou igual que $k$. Este conceito de erro é ilustrado na Figura 3. Salienta-se que, na faixa de 10 a $20 \%$ dos pontos selecionados, a diferença entre o MSE exato e o MSE estimado não é constante mas decrescente, porém, isto ocorre de forma tão lenta a ponto de sugerir que a curva é constante - o que não é o caso. Ainda que apenas os resultados relativos ao MSE entre as imagens Mandrill original e reconstruída à taxa de 0,375 bpp (QV com dicionário de tamanho 64) sejam apresentados na Figura 3, os resultados obtidos para as demais imagens testadas exibem o mesmo comportamento típico.

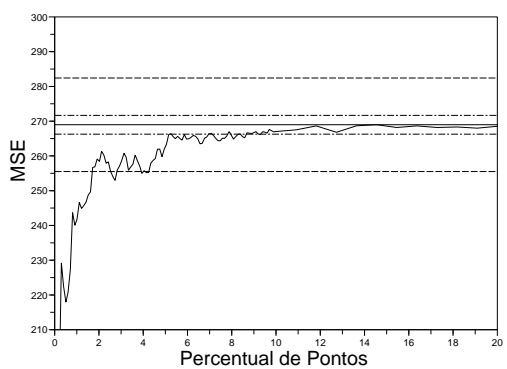

(a) Valor estimado de MSE

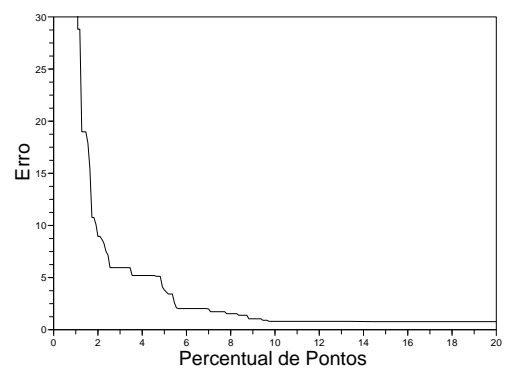

(b) Diferença entre MSE exato e estimado

Figura 3: (a) Valor estimado de MSE em função do percentual de pixels selecionados da imagem Mandrill. Considera-se QV com taxa de 0,375 bpp. A reta central, sólida, indica o valor exato da métrica (MSE calculado considerando a totalidade dos pixels das imagens original e reconstruída). As retas abaixo e acima da linha central indicam limites para erros (diferença entre MSE exato e estimado pelo percentual dos pixels) em torno de 1\% (retas próximas, desenhadas com ponto-e-traço) e $5 \%$ (retas afastadas, tracejadas). (b) Diferença entre MSE exato e MSE estimado em função do percentual de pixels selecionados.

A Tabela 1 relaciona o percentual necessário de pontos a serem amostrados na imagem Mandrill com o percentual de erro aceitável da estimativa com relação ao valor exato da métrica para cada esquema de codificação testado.

Nesta tabela, observa-se que, para obtenção de um erro de $5 \%$ foi necessário, no melhor caso (dicionário de tamanho 32) o uso de apenas 1,1\% dos pontos da 
Tabela 1: Percentual de pontos amostrados da imagem Mandrill para obter uma estimativa do valor exato da métrica dentro de cada limite de erro $(1 ; 2,5 ; 5$ e $10 \%)$. Nos parênteses ao lado de cada tamanho de dicionário, o valor exato da métrica e nos parênteses ao lado de cada percentual de pontos, o valor encontrado utilizando como estimativa aquela quantidade de pontos.

\begin{tabular}{c|c|c|c|c}
\hline Tamanho de dicion. & Erro de 1\% & Erro de 2,5\% & Erro de 5\% & Erro de 10\% \\
\hline \hline $32(311,89)$ & $64,0(311,26)$ & $4,5(310,60)$ & $1,1(301,65)$ & $1,0(295,27)$ \\
$64(268,96)$ & $9,5(271,04)$ & $5,2(266,65)$ & $1,8(265,32)$ & $1,3(259,13)$ \\
$128(229,54)$ & $56,0(229,87)$ & $8,3(227,84)$ & $1,9(218,36)$ & $1,7(218,27)$ \\
$256(195,67)$ & $49,0(195,54)$ & $8,5(193,84)$ & $4,5(186,65)$ & $1,5(183,70)$ \\
$512(154,98)$ & $42,0(156,31)$ & $2,3(155,19)$ & $1,7(153,95)$ & $0,4(149,39)$ \\
\hline
\end{tabular}

imagem. No pior caso (dicionário de tamanho 256), no entanto, foi necessário o uso de $4,5 \%$ dos pontos para obtenção da estimativa do valor exato da MSE com erro de $5 \%$. Convém salientar que, para quase totalidade das nove imagens utilizadas neste trabalho, com apenas $7 \%$ dos pixels, o erro médio quadrático calculado com o método proposto difere em menos de $5 \%$ do valor exato desta métrica. As excessões foram algumas codificações da imagem Clock (dicionários de tamanhos 64 e 128, que necessitaram, respectivamente, de 16 e $10 \%$ dos pontos para fornecer erro menor que $5 \%$ ), da imagem Lena (dicionário de tamanho 128 e necessidade de 8,9\% dos pontos), da imagem Goldhill (dicionário de tamanho 64 e necessidade de 7,9\% dos pontos) e imagem Peppers (dicionário de tamanho 32 e necessidade de $11 \%$ dos pontos), o que fornece uma economia superior a $90 \%$ no tocante ao número de operações necessárias para a obtenção da estimativa. Ressaltamos que, apesar das excessões apresentadas, do total das 45 imagens testadas para erro igual ou menor que 5\%, 29 delas necessitaram de menos de $3,5 \%$ dos pontos para fornecer uma estimativa do valor exato da métrica MSE dentro do limite tolerado de erro, enquanto que 34 delas forneceram tal estimativa com uso de $5 \%$ dos pontos da imagem.

Do ponto de vista do custo computacional de obter tais estimativas, uma vez que a sequência $V D H$ é gerada uma única vez e mantida em memória, sua contribuição no processamento da métrica é da ordem $\mathcal{O}(1)$. Em relação a sistemáticas de avaliação subjetiva, como, por exemplo, o uso de MOS [10], o método apresentado neste trabalho constitui uma alternativa para uma rápida comparação de desempenho de algoritmos de compressão de imagens, no que diz respeito à qualidade das imagens reconstruídas.

\section{Conclusão}

A avaliação da diferença métrica entre imagens é um problema relevante em processamento digital de imagens. Neste trabalho, utilizando um percentual de $7 \%$ do tamanho total da imagem, foi observado que o uso de sequências de baixa discrepância pode fornecer boas estimativas, i.e, com erro máximo igual ou inferior a 
$5 \%$ do valor exato da métrica, com evidente redução da demanda computacional, uma vez que a geração de tal sequência é um processo que somente será executado uma vez para cada tamanho de imagem. Trabalhos futuros incluem a utilização de tal procedimento em aplicações de vídeo em tempo real.

Abstract. A great challenge in image coding is the conception of efficient methods for assessing the quality of reconstructed images. This paper presents an approach for assessing the quality of images based on low discrepancy sequences. Simulation results concerning vector quantization of $256 \times 256$ images show that, with only $7 \%$ of the pixels, the mean square error calculated by the proposed approach differs less than $5 \%$ from the exact value of that measure, calculated by using the entire image.

\section{Referências}

[1] H.M. Al-Otum, Evaluation of reconstruction quality in image vector quantisation using existing and new measures, IEE Proceedings - Vision, Image and Signal Processing, 145, No. 5 (1998), 349-356.

[2] J.R. Deller Jr., J.G. Proakis, J.H.L. Hansen, "Discrete-time Processing of Speech Signals", Macmillan Publishing Co., 1993.

[3] M.P. Eckert, A.P. Bradley, Perceptual quality metrics applied to still image compression, Signal Processing, 70 (1998), 177-200.

[4] A.M. Eskicioglu, P.S. Fischer, Image quality measures and their performance, IEEE Transactions on Communications, 43, No. 12 (1995), 2959-2965.

[5] A. Gersho, R.M. Gray, "Vector Quantization and Signal Compression", Kluwer Academic Publishers, Boston, MA, 1992.

[6] R.M. Gray, Vector quantization, IEEE ASSP Magazine, (1984), 4-29.

[7] J.H. Halton, On the efficiency of certain quasi-random sequences of points in evaluating multidimensional integrals, Numer. Math., (1960), 84-90.

[8] N. Jayant, Signal Compression: technology targets and research directions, IEEE Journal on Selected Areas in Communications, 10, No. 5 (1992), 796818.

[9] L. Kuipers, H. Neiderreiter, "Uniform Distribution of Sequences", Addison Wesley Publishing Company, Inc., New York, 1992.

[10] F. Madeiro, J.M. Fechine, B.G. Aguiar Neto, M.S. Alencar, On the performance of objective quality measures for evaluating vector-quantized images, in "Proceedings of the XVII Brazilian Telecommunications Symposium (SBT'99)", Vila Velha, ES, Brazil, pp. 616-621, 1999.

[11] A. Shnayderman, A. Gusev, A.M. Eskicioglu, An SVD-Based grayscale image quality measure for local and global assessment, IEEE Transactions on Image Processing, 15, No. 2 (2006), 422-429. 
[12] K.S. Tan, P.P. Boyle, Applications of randomized low discrepancy sequences to the valuation of complex securities, Journal of Economic Dynamics and Control, (2000), 1747-1782.

[13] Van der Corput, Zahlentheoretische abschaätzungen, Math. Ann., (1921), 5379 .

[14] Z. Wang, A.C. Bovik, A universal image quality index, IEEE Signal Processing Letters, 9, No. 3 (2002), 81-84. 